\title{
Minimally invasive periodontics - A future landscape
}

\author{
Himanshu Aeran ${ }^{1 *}$, Amrinder Singh Tuli ${ }^{2}$, Megha Phogat Rana ${ }^{3}$, Basant Kaur Aulakh ${ }^{4}$, Lakshya Vishnoi ${ }^{5}$ \\ ${ }^{1}$ Director Principal, Professor and Head, ${ }^{2}$ Professor and Head, ${ }^{3}$ Professor, ${ }^{4}$ Post Graduate Student, ${ }^{5}$ Intern, Dept. of Prosthodontics, \\ ${ }^{1-4}$ Seema Dental College \& Hospital, Rishikesh, Uttarakhand, ${ }^{5} \mathrm{KGMC}$, Lucknow, Uttar Pradesh, India
}

\section{*Corresponding Author: Himanshu Aeran}

Email: drhimanu4@gmail.com

\begin{abstract}
Periodontal diseases are a heterogeneous group of diseases characterized by inflammation and the subsequent destruction of the tooth supporting tissue. Conventional periodontal regenerative procedures involving periodontal flaps for access are employed to improve shortand long-term clinical outcomes of periodontally compromised teeth. Minimally invasive therapeutic approaches with minimized incisions and trauma to the soft tissue have proven to be advantageous over the conventional therapy. This review focuses on four such techniques PerioProtect, Tri-Immuno Phasic Periodontal Therapy, Subperiosteal Minimally Invasive Aesthetic Ridge Augmentation Technique (SMART) and Flapless technique for Bone Graft. PerioProtect is a non-invasive chemical debriding therapy that involves a customized tray for patients to help manage biofilms, growing in the spaces or pockets between teeth and gum tissue. Tri-immuno phasic therapy is minimally invasive technique, by William Hoisington, which allows clinician to go under the gum line and destroy anaerobic bacteria non-surgically. SMART was suggested by Kent et al. in which a small vertical incision is made in the alveolar ridge and bone graft injected under the periodontium. Flapless technique for bone graft is a non-invasive technique which involves scaling and root planing, and insertion of single grains of bone mineral through the crevicular space into the pocket until it is completely filled. These minimally invasive procedures offer an efficient, safe less traumatic alternative in the treatment of periodontitis.
\end{abstract}

Keywords: PerioProtect, Tri-Immuno, Occlusal forces.

\section{Introduction}

Periodontitis can be defined as the inflammation of the supporting tissues of the teeth usually a progressively destructive change leading to loss of bone and periodontal ligament.

It requires meticulous attention by the clinician, dental hygienist and great efforts by the patient in the form of compliance. There are considerable variations in clinical presentations of periodontal disease, which have been spoken as disease activity models. Although plaque/biofilm is the principal causative factor, several other host-related, environmentally altered factors seem to be playing at tandem. Control of plaque by mechanical means and use of antimicrobials locally and systemically have been the principal methods of disease prevention and containment. However, mechanical plaque control is tedium and taxing on the patients resources and not many patients have the inclination or mindset to scrupulously adhere to demanding and exacting oral hygiene practices. Use of antimicrobials has its own limitations in the form of return and recolonization of the organisms once there is cessation of antibiotic use and other issues related to their prolonged use. ${ }^{1}$

Advances in periodontal science and practice over the last decade have radically changed the understanding of periodontal diseases and have opened new, exciting prospects for both non-surgical and surgical therapy of periodontal diseases. Mechanical methods of subgingival debridement accomplished by thorough scaling and root planning, accompanied by oral hygiene procedures, have served as the gold standard of periodontal therapy for decades. ${ }^{2}$

Various treatment options are available in the armory of a periodontist including surgical and non- surgical therapy. Not satisfied with traditional weapons to combat oral microbiota and restore lost alveolar bone support, ingenious clinicians, scientist and researchers have turned novel and sometimes exotic avenues and explored new frontiers of physical laws, pharmacological molecules and new concepts as available for other medical situations. The newer trends are-

1. PerioProtect

2. Tri-immunophasic periodontal therapy (TIP)

3. S.M.A.R.T

4. Flapless technique for bone graft

\section{Perioprotect $^{3}$}

PerioProtect is a comprehensive method that is customized for individual patients to help manage biofilms, growing in the spaces or pockets between teeth and gum tissue. The overall goal of the Perio Protect Method is to manage oral biofilm with minimally invasive dentistry for lasting oral health.

The method is a combination of treatments, including a non-invasive chemical debriding therapy used in conjunction with traditional mechanical debriding procedures. The chemical therapy involves a tray delivery of doctorprescribed solutions to chemically debride biofilm from the periodontal pocket and alter the pocket's microbiological environment to disrupt biofilm growth.

\section{Mechanism}

The use of non-specific chemical agents to kill all of the planktonic cells and some of the biofilm cells, in a particular ecosystem. This strategy, which has proven to be very successful in industrial applications and in the protection of various catheters from bacterial colonization and consequent infection, depends on the alteration of the microbial ecology of an ecosystem so that biofilm formation is minimized. Biofilm bacteria show the same susceptibility to non-specific 
oxidizing agents as their planktonic counterparts, so that industrial biocides and "catheter lock" solutions kill all of the planktonic cells and as many of the biofilms cells as their stochiometry allows. The regular application of $0.5 \%$ bleach in the "Y" sets used to protect Tenchoff catheters from colonization and infection has been successful for several years, in spite of the extreme susceptibility of the peritoneum to bacterial incursions.

The PerioProtect therapeutic system uses this concept, because it delivers peroxide and an antioxidant to the periodontal space at regular intervals and kills the planktonic bacteria and enough of the biofilm bacteria to gradually alter the microbial ecology of this ecosystem. The direct result of this alteration in the microbial ecology of the periodontal space is a sharp reduction in the rate at which available surfaces are colonized; the relevant measurement is the rate of colonization of an inert carrier material introduced into the area for a specified length of time. This measurement is clinically relevant because it is the inflammatory response of the gingival tissues to the presence of planktonic and detached biofilm cells that lies at the base of the etiology of this, and all other, chronic biofilm infections.

\section{Advantages}

1. Pain free

2. Reduces need for surgery

3. Easy to wear

A proof of concept study ${ }^{4}$ was done to determine the efficacy of a custom-fabricated tray in placing antimicrobial and debriding agents in the periodontal pockets of persons with active gingival infections. The ability of this tray is to maintain sufficient concentrations of medication in the periodontal pockets to have a therapeutic effect is evaluated here with theoretical modeling and practical application. This study indicates that the custom-formed prescription Perio Tray can place and maintain medications in periodontal pockets at a sufficient concentration for the medication to have a significant therapeutic effect.

A study done by Mark S. Putt ${ }^{5}$ in 2012, showed that, adjunctive use over three months of $1.7 \%$ hydrogen peroxide gel, locally administered using PerioProtect in the treatment of subjects with moderate to advanced periodontitis, demonstrated statistically significant clinical improvements in pocket depths and bleeding when compared with SRP alone.

Another study by Duane C. Keller ${ }^{6}$ in 2017, showed that, direct medication deliveries of hydrogen peroxide, oxygen and Vibramycin into the periodontal pocket with a PerioTray are effective in controlling the biofilm responsible for periodontal disease.

\section{Tri-immunophasic periodontal therapy ${ }^{7-9}$}

US periodontal therapist William Hoisington has commenced a new technique in treating periodontal disease which solves the issue of periodontium in an entirely new way. TriImmuno - Phasic periodontal therapy, otherwise known as TIP, allows practitioners to go up to the crest of the alveolar bone and destroy anaerobic bacteria - aggressive pathogens which not only causes degeneration of alveolar bone and gingiva, but also penetrate the body's circulation and contribute to systemic complications such as coronary heart disease, osteoporosis, pre term low birth weight and infertility.

TIP periodontal therapy methods are:

1. Bone One Session Treatment

2. Controlling Occlusal Forces

3. Oral Hygiene reinforcement with adjuvant modalities

4. Life Style modification

5. Enhancing nutrition and exercise

\section{Hypothesis}

The tri-immuno-phasic periodontal therapy, hypothesized that the body is capable of heal the periodontium as similar to that of the other forms of repair. When appropriate conditions are achieved damaged periodontal bone can heal as like other bones in the human body.

\section{Immune system phases}

TIP periodontal therapy influences the local and personal factors which modify periodontal disease progression and improve the condition for healing by aiding the body in all three immune phases. The three immune phases are: vigilant readiness, defense phase, repair and regeneration.

\section{Bone One Session Treatment (BOST)}

Bone one session treatment (BOST) is an aerobic treatment that eliminates periodontal disease in the deepest pockets and supporting alveolar bone. A typical BOST treatment of full mouth takes 4 to 5 hours during one day period. BOST minimize damage to the gingiva, bone, and periodontal apparatus during treatment. It uses technique called stretch flap. This stretch flap technique allows access all the way down to the deepest areas of the roots and the surface of the bone, without giving incision.

\section{Stretch flap technique}

First step: In this step universal 4R-4L curette is inserted inside the sulcus, where working end facing towards tooth surface and blunt non-working end facing tissue. A slight pressure is applied on tissue to begin stretching while removing superficial plaque and calculus.

Second step: The direction changes to a circumferential motion starting at the corner to mobilize the tissue and avoid pulling the papilla free and in essence creating an incisional flap.

Third step: Here the curettes are inverted to allow the rounded tip of the curettes to plasty the surface of bone and remove any attached granulation tissue or degenerated attachment. The goal is to achieve smooth, regular bone surface and fresh bleeding to flush out bacteria and toxins from the porosities.

\section{Healing after BOST}

After the bone one session treatment the clot that is firmly attached to the clean bone serves as a scaffold. The stem cells 
can move on along it and up the root surfaces at the rate of $0.05 \mathrm{~mm}$ per day for eight days and thicken the layer on the clot. As healing time increases, the pockets gradually fill from the bottom with very dense, partially mineralized connective tissue in a time period of 4 to 6 weeks and finally it will become acellular. Once the new mineralized acellular connective tissue attachment is in place is in about one month, the bone naturally heals under it. A new dense layer of cortical bone forms over the healed inner (cancellous) bone in about eight more months.

\section{Controlling the Occlusal Forces}

Objectives: Controlling the Occlusal forces, prevention of Tooth mobility and further tissue damage through coronoplasty and splinting.

With weekend periodontium normal occlusal forces become traumatic and lead to shift of bite. Treatment includes enameloplasty, coronoplasty, and occlusal splint will redistribute the forces among all the teeth.

\section{New Oral Hygiene Routine}

Brushing, flossing, and other conventional methods of dental hygiene used to maintain oral hygiene. Aerobic oral hygiene kit (Perio-aid) that is created specifically to clean the area under gingiva and eliminate the disease-causing anaerobic bacteria in deep pockets and into root concavities where brush and floss cannot reach.

\section{Life Style, Nutrition and Exercise}

Eat balanced diet by eating regular meals, avoid of excessive sugar, avoid of excessive snacks, avoid granular food like nuts, seeds, chips, whole grain breads etc. Increase intake of vitamins and minerals. Vitamin $\mathrm{c}$ and zinc are most important. Avoid smoking, smoking reduces circulation, smoking depresses certain immune cells. Exercise should be regular because its increases circulation to bring in building blocks and oxygen to the tissues as well as the vitamins and minerals that permit proper uptake.

Step wise recovery process of tip

Day 1: Bost treatment

Day 2: Patient restart oral hygine

Day 3: Bleeding stop

Day 4: Pockets start to fill in

Day 5: Inflammation continues to receed

Day 8: Pocket sealing up

Day 14: Resume normal chewing

Day 15: Check up

Day 30: Pockets have filled in

Day 35: Esthetics restoration can start.

Day 45: Bone is beginning to heal

3 months: Check -up

9 months: Bone completely healed

\section{Advantages of TIP}

1. Performed without invasive surgery

2. No incisions and sutures mean less discomfort

3. Hygiene starts the next day

4. Preserves esthetics and minimized sensitivity
5. Fast recovery from inflammation

6. No necrosis of bone

7. No gingival damage

8. Faster reliable

9. Less complication

10. Patience compliance is good

\section{S.M.A.R.T $\mathbf{T}^{10,11}$}

The Subperiosteal Minimally invasive Aesthetic Ridge Augmentation technique (SMART) was developed as an alternative to traditional guided bone regeneration procedure.

\section{Procedure}

Presurgical dental prophylaxis is done. Local anesthesia is achieved. One or more full thickness incisions are made in areas remotely requiring bone grafting. These incisions are kept away from the gingival margin and sulcus. A BardParker blade is used to cut through the gingiva, oral mucosa and periosteum until contact with bon is made. Specially designed instrument are used to carefully elevate the full thickness of the mucosa with an intact periosteum attached to the surface. Mucoperiosteum elevation proceeded in a tunnellike fashion until the target site for bone augmentation is accessed. A subperiosteal pouch is then created that could accommodate an adequate extension and volume of the bone graft material. Bone graft is delivered and manipulated through the tunnel access until the material reaches the defect area. Specialized instruments are used to compact and shape the material. Primary closure of the vertical incisions is achieved.

\section{Advantage}

1. Minimal or no swelling

2. Increased predictability

3. Consistent augmented bone volumes

4. No membranes, screws or decortication

5. Less bleeding

6. Less discomfort

7. Less surgery recovery time

8. Less technique sensitive

9. Better preservation of keratinized gingiva

\section{Flapless technique for bone graft $^{12,13}$}

The efficacy of grafts in treatment of deep pockets and regeneration or repair of lost alveolar bone is well established by clinical studies, and the surgical techniques used for such grafts are, however, quite critical. Most grafts involve the incision of the soft tissue and the insertion of the graft material, but the resulting flap of primary soft tissue must then be sutured so it covers the graft. When the soft tissues overlying a graft do not completely cover the inserted material, contraction occurs and the graft material is exposed to exfoliation, as well as to contamination of the graft site. Various types of flap have been proposed in order to provide better coverage of the graft, including mucoperiosteal flap, modified Widman flap, papilla preservation flap, variation of papilla preservation flap, and minimally invasive surgery. 
A flapless technique for graft placement was designed to overcome the risk of contraction. After great scaling and root planing, the bone graft material was inserted through the crevicular space into the pocket, grain by grain using light pressure with the help of spatula. There was no cutting of the soft tissue, thus, avoiding all of the problems caused by contamination and exfoliation of graft. Moreover, the gingival covering provides irrigation with blood, which leads to the rapid repair of the tissues. Furthermore, since there is no incision, the papilla is maintained intact and little retraction occurs; the clinical attachment level thus can improve significantly, and the alveolar osseous crest can recover.

\section{Advantages \\ 1. Fast \\ 2. Less aggressive \\ 3. No contamination}

\section{Conclusion}

These minimally invasive procedures offer an efficient, safe less traumatic alternative in the treatment of periodontitis.

\section{Source of funding}

None.

\section{Conflict of interest}

None.

\section{References}

1. Kumar JA, Anumala N, Avula H. Novel and often bizarre strategies in the treatment of periodontal disease. J Indian Soc Periodontol 2012;16:4-10.

2. Kumar KN, Chandhra MP, Babu R, Srikanth C, Paul AR. New trends in periodontics. J Evol Med Dent Sci 2012 p. 546-558.

3. Dunlap T, Duane KC. Subgingival delivery of oral debriding agents: A proof of concept. J Clin Dent 2011;22:149-58.

4. Putt MS, Proskin HM. Custom Tray Application of peroxide gel as an scaling and root planng in the treatment of perioontitis:A randomized, controlled three-month Clinical Triad. J Clin Den 2012;23:48-56.

5. Keller DC and Buechel M. Direct medication delivery modifies the periodontal biofilm. Oral Biol Dent 2017;5:1.

6. Dr. Kumar YP, Dr. Lalaivani V, Dr. Rajapandian K, Dr. Malakar M. Tri-immuno phasic periodontal therapy. World $J$ Pharm Res 2016;5(9): 356-60.

7. Hoisington W. New developments in perio: Tri-immunophasic therapy. Prev Dent 2006;1(2):30-34.

8. Arpita R, Swetha JL, Babu MR, Sudhir R, Recent trends in non-surgical periodontal care for the general dentist $-\mathrm{A}$ review. Bangle J Dent Res Edu 2012;4(2):78-82.

9. Lee EA. Subperiosteal minimally invasive aesthetic ridge augmentation technique (SMART): A new standard for bone reconstruction of the jaws. Int J Periodontics Restorative Dent 2017;37(2):165-73

10. Hyun SK, Young KK, Pil YY. Minimal invasive horizontal ridge augmentation using subperiosteal tunneling technique. Maxillofac Plast Reconstr Surg 2016: 38-41.

11. Argento MA, Manara LRB, Berni VC, Cortelazzo AL. Flapless technique for periodontal bone grafts in treatment of severe periodontitis. Presentation and long-term retrospective study. J Microbial Biochem Technol 2010;2:107-10.
12. Fugazzotto PA. Maintaining primary closure after guided bone regeneration procedures: introduction of new flap design and preliminary results. J Periodontol 2006;7:1452-7.

13. Kenney EB, Lekovic V, Han T, Carranza FA Jr, Dimitrijevic B. The use of solid implants in periodontal bony defects. I. Clinical results after six months. J Periodontol 1985;56:82-7.

How to cite this article: Aeran $\mathrm{H}$, Tuli AS, Rana MP, Aulakh BK, Vishnoi L. Minimally invasive periodontics - A future landscape. Int J Oral Health Dent 2019;5(4):180-3. 